\title{
Risco teratogênico: a percepção em diferentes segmentos da população
}

\author{
Emília Katiane Embiruçu ${ }^{I}$ \\ Ney Boa Sorte ${ }^{2}$ \\ Renata Vidal \\ Licemary Lessa ${ }^{3}$ \\ Eliana Panão ${ }^{3}$ \\ Ana Carolina Mota ${ }^{3}$ \\ Tirza Santana ${ }^{3}$ \\ Indira Araújo ${ }^{3}$ \\ Maria Betânia Toralles ${ }^{4}$ \\ Angelina Xavier Acosta ${ }^{4}$
}

\section{Resumo}

A percepção errônea do real risco teratogênico das drogas às quais as gestantes são expostas pode induzir a erros graves de conduta perante uma gravidez, ou pela interrupção desnecessária da gestação desejada, ou devido às seqüelas permanentes nas crianças. O conhecimento sobre o risco teratogênico é de fundamental importância para a população geral e, principalmente, para a comunidade médica. A percepção do risco teratogênico de quinze fármacos habituais e duas drogas ilícitas foi avaliada entre médicos, estudantes de medicina e mulheres leigas, utilizando-se uma escala visual, na qual os entrevistados assinalaram o risco em percentual de uma criança nascer com anomalia congênita secundária à exposição intra-útero às drogas estudadas. A média da percepção do risco teratogênico para as drogas avaliadas foi maior do que o risco real entre todos os grupos estudados. O risco real de anomalia congênita na população geral foi melhor reconhecido entre os médicos ginecologistas e obstetras. O melhor índice de acerto foi para drogas mais rotineiramente utilizadas durante a gestação (paracetamol e amoxacilina), de acordo com os obstetras.

Palavras-chave: risco teratogênico; gestação; anomalia congênita.

\section{INTRODUÇÃO}

Os teratógenos constituem agentes ambientais, químicos, físicos e biológicos, que podem causar anormalidades obstétricas e (ou) fetais. A partir da tragédia da talidomida na década de sessenta, sobre cada droga nova lançada no mercado levantam-se suspeitas de tratar-se de uma "nova talidomida". Atualmente, menos de trinta drogas são reconhecidas como potencialmente teratogênicas e possuem malformações características associadas, mas

\footnotetext{
${ }^{1}$ Professora de Neuroanatomia e Neuropatologia. Laboratório de Anatomia Humana. UNEB. Salvador - BA

${ }^{2}$ Professor Assistente da Faculdade Baiana de Medicina e Saúde Pública. FBDC. Salvador - BA

${ }^{3}$ Acadêmico do Curso de Medicina. UFBA. Salvador - BA

${ }^{4}$ Doutora em Medicina Interna. Professora Adjunto do Departamento de Pediatria. UFBA. Salvador - BA
}

Correspondência para / Correspondence to:

Emília Katiane Embiruçu

Departamento de Ciências da Vida. Universidade do Estado da Bahia

Campus I. Rua Silveira Martins, s/n - Cabula.

41.195-001 Salvador - Bahia - Brasil.

E-mail: ekeleao@ig.com.br. ekeleao@yahoo.com.br. 
nenhuma delas com risco de efeito teratogênico próximo a $100 \%$. (JASPER et al., 2001)

Os estudos que avaliam a segurança ou riscos das drogas devem ser analisados com cautela por parte da classe médica. A interpretação inadequada desses resultados pode levar a condutas ou aconselhamentos errôneos sobre os efeitos deletérios fetais (KOREN; PASTUSZAK; ITO, 1998; BATINO, 2001; CEFALO, 2001; SANZ; GOMEZ-LOPEZ; MARTINEZQUINTAS, 2001; KOREN; LEVICHECK, 2002).

A exposição a agentes teratogênicos muitas vezes ocorre de maneira inadvertida no início da gestação, visto que, nesse período, as mulheres podem não ter conhecimento da gravidez. Esse acontecimento, associado à percepção errônea do risco teratogênico, pode induzir a uma interrupção desnecessária da gestação. Por outro lado, a manutenção da exposição a um agente comprovadamente teratogênico ou a doença materna sem tratamento adequado pode produzir sérios danos à saúde fetal (KOREN, 2001).

A percepção correta dos riscos desses agentes teratogênicos está limitada a profissionais que atuam em serviços especializados em teratogênese, o que justifica a necessidade de difusão dessas informaçōes para a população em geral, principalmente à classe médica, que se configura como o veículo mais efetivo na propagação de tais conhecimentos (POLIFKA; FAUSTMAN; NEIL, 1997).

No Brasil, os estudos sobre teratologia são escassos. O objetivo deste trabalho foi avaliar o conhecimento de médicos, estudantes de medicina e mulheres a respeito do uso de quinze fármacos e duas drogas ilícitas (álcool e cocaína) durante a gestação, utilizando uma escala visual. Durante as entrevistas, foram promovidas informações sobre as drogas questionadas na pesquisa.

\section{MATERIAIS E MÉTODOS}

Entre outubro de 2002 e junho de 2003, após explicação dos objetivos da pesquisa e assinado o consentimento informado, foi aplicada uma ficha-padrão com identificação, idade e uma escala visual, testada anteriormente por Sanz, Gomez-Lopez e Martinez-Quintas (2001), na qual cada participante assinalava o risco estimado de indução de efeitos teratogênicos correspondentes às drogas em estudo: álcool, amoxicilina, aspirina, benzodiazepínicos, carbamazepina, cocaína, eritromicina, etretinato, fenitoína, haloperidol, metoclopramida, misoprostol, paracetamol, ranitidina, talidomida e warfarin, além do risco estimado na população geral. Também foi avaliado o conhecimento prévio ou utilização dos serviços prestados pelo Serviço de Informações sobre Agentes Teratogênicos da Bahia (SIAT-BA). Aos entrevistados que não conheciam determinada droga pelo nome químico forneceram-se os nomes comerciais e as indicações das drogas. O entendimento do risco era assinalado com um ponto na linha vertical não graduada, onde o menor risco $(0 \%)$ era representado pela extremidade inferior da linha e o maior risco $(100 \%)$ na extremidade superior, sendo permitida a marcação para cada droga em qualquer local da escala.

Imediatamente após a aplicação do questionário, foi fornecida aos entrevistados uma apostila com uma tabela das drogas envolvidas, a farmacologia, a teratogenicidade em animais e (ou) seres humanos e a conclusão a respeito do potencial teratogênico correspondente, informações compiladas de bancos de dados computadorizados e bibliografia biomédica especializada (SCHARDEIN, 2000; SANSEVERINO; SPRITZER; SCHÜLER-FACCINI, 2001), e expressas em linguagem acessível a todas as pessoas.

A amostra estudada foi formada por grupos de estudantes de medicina iniciantes (26) e concluintes (20), médicos clínicos gerais (19) ginecologistas e obstetras (10), profissionais do setor público, privado ou ambos, e mulheres gestantes (32) e não-gestantes (40). Os participantes foram escolhidos de forma aleatória, através de busca ativa em locais de referência: universidades, clínicas, hospitais e shopping centers, respectivamente.

Os resultados foram avaliados separadamente para cada grupo de participantes, e para cada medicação. $\mathrm{O}$ valor da percepção de cada indivíduo foi obtido através da mensuração da diferença, em centímetros, entre o ponto de menor risco $(0 \%)$ e o ponto marcado pelo en- 
trevistado. Então, aplicou-se uma regra de três simples, que resultou no valor estimado do risco para cada medicação.

O valor da média e do desvio-padrão, além da mediana, foi calculado para cada medicação, em cada grupo analisado. Devido ao número de componentes finais em cada grupo e das respostas não terem uma distribuição normal, utilizou-se o teste de Mann-Whitney para comparação de dois grupos e o teste de KruskalWallis, para comparação em três ou mais grupos. Calculou-se o índice de acerto para cada medicação em cada grupo estudado. Para comparação dos percentuais de acerto para cada droga entre os grupos, utilizou-se o qui-quadrado.

\section{RESULTADOS}

Da amostra, 29 pessoas (19,7\%) conheciam o Serviço de Informaçōes sobre Agentes Teratogênicos (SIAT), mas somente 3 entrevistados $(2,0 \%)$ já haviam utilizado o serviço para consulta, sendo uma gestante, um obstetra e um clínico geral.

A média da percepção do risco de teratogênese entre os grupos pesquisados para as medicações propostas foi acima do risco real estimado na literatura, independentemente do grupo ou da medicação analisada (FIGURA 1). A percepção real de risco para teratogênese, que é estimada em até 5\%, foi referida com correção entre $40 \%$ dos obstetras (melhor acerto), em $5,3 \%$ dos clínicos (pior acerto) e foi de 10\%, $15,4 \%, 21,9 \%$ e $17,5 \%$ entre os concluintes, os estudantes iniciantes, as gestantes e as não gestantes respectivamente.

$\mathrm{O}$ melhor índice de acerto, entre os obstetras, para a real percepção de risco de teratogênese foi para o paracetamol, correspondendo a $80 \%$, seguido da amoxacilina (50\%). Nesse mesmo grupo, menos de $10 \%$ acertaram o risco real para a eritromicina e haloperidol, e nenhum acerto foi verificado para a talidomida, carbamazepina, cocaína, fenitoína e misoprostol.

Entre os clínicos gerais, o melhor índice de acerto ocorreu para o álcool $(35,8 \%)$, seguido da amoxacilina $(26,3 \%)$, da aspirina, do paracetamol $(21,1 \%)$ e do etretinato e da ranitidina $(15,8 \%)$. Não houve acerto, nesse grupo, para a fenitoína, a talidomida, os benzodiazepínicos, a cocaína e o misoprostol, e as outras drogas avaliadas tiveram acerto menor que $10 \%$. O percentual de acerto dos demais grupos está demonstrado na Tabela 1 .

Tabela 1 - Percentual de acerto do risco real de efeito teratogênico entre os grupos analisados.

\begin{tabular}{|c|c|c|c|c|c|c|c|}
\hline Média & $\begin{array}{c}\text { Risco Real } \\
(\%)\end{array}$ & $\begin{array}{c}\text { Iniciantes } \\
(\%)\end{array}$ & $\begin{array}{c}\text { Concluintes } \\
(\%)\end{array}$ & $\begin{array}{l}\text { Clínicos } \\
\text { Gerais(\%) }\end{array}$ & $\begin{array}{c}\text { Gineco- } \\
\text { obstetras (\%) }\end{array}$ & $\begin{array}{c}\text { Gestantes } \\
(\%)\end{array}$ & $\begin{array}{c}\text { Não Gestantes } \\
(\%)\end{array}$ \\
\hline Geral & $<5$ & 15,4 & 10,0 & 5,3 & 40,0 & 21,9 & 17,5 \\
\hline Álcool & $<33$ & 15,4 & 35,0 & 36,8 & 20,0 & 40,6 & 12,5 \\
\hline Amoxicilina & $<5$ & 23,1 & $0^{* * 2 x}$ & 26,3 & 50,0 & 15,6 & $5,0^{* *}$ \\
\hline Aspirina & $<5$ & 19,2 & 25,0 & 21,1 & 30,0 & 21,9 & 12,5 \\
\hline Benzodiazepínico & $<5$ & 7,7 & $0^{*}$ & 0 & 30,0 & $0^{*}$ & $2,5^{*}$ \\
\hline Carbamazepina & $<5$ & 7,7 & 0 & 5,3 & 0 & 6,3 & 2,5 \\
\hline Clorpromazina & $<5$ & 3,8 & 0 & 5,3 & 20,0 & 6,3 & 7,5 \\
\hline Cocaína & $<15$ & 7,7 & 0 & 0 & 0 & 6,3 & 2,5 \\
\hline Eritromicina & $<5$ & 11,5 & 5,0 & 10,5 & 10,0 & 6,3 & 5,0 \\
\hline Etretinato & $16-30$ & 7,7 & 25,0 & 15,8 & 20,0 & 9,4 & 12,5 \\
\hline Fenitoína & $\leq 10$ & 0 & 5,0 & 0 & 0 & 0 & 5,0 \\
\hline Haloperidol & $<5$ & 3,8 & 0 & 5,3 & 10,0 & 6,3 & 2,5 \\
\hline Metoclopramida & $<5$ & 11,5 & 10,0 & 10,5 & 40,0 & 37,5 & 20,0 \\
\hline Misoprostol & $<10$ & 7,7 & 5,0 & 0 & 0 & 6,3 & 0 \\
\hline Paracetamol & $<5$ & $23,1^{* *}$ & $15,0^{* *}$ & $21,1^{* *}$ & 80,0 & 40,6 & $17,5^{* * *}$ \\
\hline Ranitidina & $<5$ & 7,7 & 10,0 & 15,8 & 20,0 & 6,3 & 12,5 \\
\hline Talidomida & $11-35$ & 0 & 10,0 & 0 & 0 & 9,4 & 10,0 \\
\hline Warfarin & $6-25$ & 23,1 & 25,0 & 0 & 10,0 & 12,5 & 5,0 \\
\hline
\end{tabular}

Notas: \# Representa diferenças entre Iniciantes e Concluintes. \# $\mathrm{p}<0.05$; \#\# $\mathrm{p}<0.01$; \#\# $\mathrm{p}<0.001$.

$\&$ Representa diferenças entre Concluintes e Clínicos. \& $\mathrm{p}<0.05 ; \& \& \mathrm{p}<0.01 ; \& \& \& \mathrm{p}<0.001$.

$\$$ Representa diferenças entre Gestantes e Não gestantes. \$ $<<0.05 ; \$ \$ p<0.01 ; \$ \$ \$ p<0.001$.

* Representa diferenças entre Gineco-obstetras e os outros grupos. ${ }^{*} \mathrm{p}<0.05 ;{ }^{* *} \mathrm{p}<0.01 ;{ }^{* * *} \mathrm{p}<0.001$. 
Em relação à percepção do risco de teratogênese entre os grupos analisados, houve diferenças entre os grupos (TABELA 2). As mulheres não gestantes perceberam um maior risco de anomalias fetais em relação às gestantes para a metroclopramida $(p=0,04)$ e o paracetamol $(p<0,001)$. Apesar de outras medicaçôes serem percebidas como de maior risco pelas não gestantes, não houve significância. Contudo, para a cocaína, houve uma maior atribuição de risco entre as gestantes, mesmo não havendo significância estatística $(\mathrm{p}=0,053)$.

Tabela 2 - Valores da média da "percepção do risco de teratogênese" entre os grupos pesquisados.

\begin{tabular}{|c|c|c|c|c|c|c|c|}
\hline Média & Risco Real & Iniciantes & Concluintes & $\begin{array}{c}\text { Clínicos } \\
\text { Gerais }\end{array}$ & $\begin{array}{l}\text { Gineco- } \\
\text { obstetras }\end{array}$ & Gestantes & $\begin{array}{c}\text { Não } \\
\text { Gestantes }\end{array}$ \\
\hline Geral & $<5$ & 21,26 & 20,67 & 14,71 & 14,94 & 28,04 & 29,21 \\
\hline Álcool & $<33$ & 42,5 & $62,34^{\# * *}$ & 54,23 & 51,4 & 54,13 & $71,56^{*}$ \\
\hline Amoxicilina & $<5$ & 36 & $30,79^{*}$ & $14,15^{\text {secexect }}$ & 17,87 & $44,68^{*}$ & $54,78^{\cdots \cdots *}$ \\
\hline Aspirina & $<5$ & 19,68 & 25,02 & 24,28 & 39,33 & 30,02 & 35,37 \\
\hline Benzodiazepínico & $<5$ & $48,26^{*}$ & $51,05^{* * *}$ & $47,93^{* * *}$ & 22,97 & $67,52^{* * a x}$ & $64,75^{* \times * *}$ \\
\hline Carbamazepina & $<5$ & 49,23 & 49,61 & 45,9 & 51,75 & 65,38 & 67,13 \\
\hline Clorpromazina & $<5$ & 47,78 & 48,36 & 37,71 & 36,85 & 49,13 & 59,56 \\
\hline Cocaína & $<15$ & 78,31 & 72,28 & 74,56 & 72,33 & $87,42^{*}$ & $91,74^{* *}$ \\
\hline Eritromicina & $<5$ & 39,81 & 46,84 & 33,81 & 39,6 & 48,57 & 62,55 \\
\hline Etretinato & $16-30$ & 34,02 & $59,08^{\# * *}$ & $69,53^{808 x}$ & 72,65 & $39,29^{* *}$ & $38,88^{*}$ \\
\hline Fenitoína & $\leq 10$ & 56,07 & 54,06 & 46,62 & 49,81 & 70,14 & 69,65 \\
\hline Haloperidol & $<5$ & 61,97 & 47,13 & $43,95^{8 x}$ & 41,28 & $68,47^{*}$ & $73,75^{* *}$ \\
\hline Metoclopramida & $<5$ & $30,1^{*}$ & 47,17 & 28,51 & 19,57 & 23,91 & $40,07^{\$}$ \\
\hline Misoprostol & $<10$ & 79,42 & 63,94 & 65,58 & 58,59 & $87,83^{* *}$ & $91,21^{\text {wax }}$ \\
\hline Paracetamol & $<5$ & $21,52^{*}$ & $15,52^{* * *}$ & $19,24^{* * *}$ & 3,7 & $13,54^{*}$ & $29,28^{\$ \$ \$ \$ * x}$ \\
\hline Ranitidina & $<5$ & 42,1 & $32,08^{*}$ & $24,18^{8 x}$ & 22,12 & $52,98^{* *}$ & $60,73^{\ldots+k}$ \\
\hline Talidomida & $11-35$ & 77,81 & $91,39^{*}$ & $94,39^{8 x}$ & 92,36 & $58,8^{* * *}$ & $61,92^{* * *}$ \\
\hline Warfarin & $6-25$ & 46,63 & 44,55 & 56,49 & 63,11 & 53,93 & 61,67 \\
\hline
\end{tabular}

Notas: \# Representa diferenças entre Iniciantes e Concluintes. \# $\mathrm{p}<0.05$; \#\# $\mathrm{p}<0.01$; \#\# $\mathrm{p}<0.001$.

\& Representa diferenças entre Concluintes e Clínicos. \& $\mathrm{p}<0.05$; \& \& $\mathrm{p}<0.01$; \& \& \& $\mathrm{p}<0.001$.

\$ Representa diferenças entre Gestantes e Não gestantes. $\$ \mathrm{p}<0.05 ; \$ \$ \mathrm{p}<0.01 ; \$ \$ \$ \mathrm{p}<0.001$.

* Representa diferenças entre Gineco-obstetras e os outros grupos. ${ }^{*} \mathrm{p}<0.05 ;{ }^{* *} \mathrm{p}<0.01 ;{ }^{* * *} \mathrm{p}<0.001$.

Comparando-se os grupos de estudantes, os concluintes apresentaram uma percepção de risco maior que os iniciantes para o álcool, o etretinato e a talidomida. Comparando-se esse resultado com os dos obstetras, observa-se que os concluintes atribuem um maior risco de teratogênese para a amoxacilina, benzodiazepínicos, paracetamol e ranitidina, e um menor risco para o etretinato. Quando se analisa a percepção de risco dentro da faixa descrita na literatura, houve uma melhor percepção para a amoxacilina $\left(c^{2}=5,308 ; p=0,021\right)$ entre os iniciantes. Apesar de não ter sido significante, alunos concluintes tiveram mais acertos para a talidomida e o etretinato, drogas sabidamente com maior efeito teratogênico.
Ao se compararem os dois grupos de médicos (clínicos e obstetras), para o paracetamol e os benzodiazepínicos houve diferenças significativas na percepção do risco de teratogênese ( $\mathrm{p}=0,02$ e $\mathrm{p}=0,012$; respectivamente), sendo o risco atribuído pelos obstetras menor para as duas medicações.

Comparando os obstetras com as gestantes, a percepção do risco de teratogênese é maior entre as gestantes para a maioria das medicações, exceto para a aspirina, warfarin, etretinato e talidomida, sendo estas duas com significância.

\section{DISCUSSÃO}

Neste trabalho, a metodologia aplicada foi a mesma empregada por Sanz, Gomez-Lopez 
e Martinez-Quintas (2001) em estudo semelhante em que quatorze drogas foram questionadas. De forma geral, observamos que as percentagens de acertos (TABELA 1) foram baixas, e as médias de percepção dos riscos superestimadas, o que atribuímos, em parte, ao instrumento utilizado, visto que as pessoas tendem a marcar pontos próximos ao centro da escala, o que dificulta uma representação acurada dos valores menores, como foi salientado no estudo anterior. Ao mesmo tempo, fomos rígidos ao considerarmos os acertos.

A distribuição das médias (FIGURA 1) dos riscos percebidos foi ampla, demonstrando grande heterogeneidade dos resultados entre os participantes. O paracetamol foi a droga que apresentou uma menor dispersão e a média mais próxima do risco real. Acreditamos que isso se deve ao fato de essa medicação ser amplamente utilizada em nosso meio para tratamento de sintomas comuns, como observado em uma coorte de 1.529 grávidas com prevalência de uso dessa substância de 41\% (STREISSGUTH et al., 1987).

As drogas relacionadas a anomalias congênitas previamente conhecidas e atribuídas aos efeitos teratogênicos foram as que tiveram as médias de risco percebidas como superestimadas entre todos os grupos, principalmente a talidomida, a qual está associada à tragédia supracitada.

Os ginecologistas e obstetras apresentaram altas taxas de acerto para o risco geral da população e, entre as drogas questionadas, para as mais freqüentemente utilizadas na gestação: metoclopramida, amoxicilina, aspirina, benzodiazepínico e, principalmente, paracetamol. As gestantes tiveram as maiores taxas de acerto para os mesmos itens que os ginecologistas e obstetras, exceto o benzodiazepínico e o álcool. Atribuímos esse resultado à influência dos médicos assistentes, cujas informações podem ter sido transmitidas através do pré-natal, o que salienta a sua importância. No entanto, a taxa de acerto das gestantes para o benzodiazepínico foi menor e a do álcool foi maior quando comparada ao grupo dos referidos médicos. O álcool freqüentemente é citado nos meios de comunicação mais populares como prejudicial ao feto, enquanto que os benzodiazepínicos são referidos basicamente na literatura científica.

$\mathrm{O}$ uso freqüente de medicações como o paracetamol e a metroclopramida, muitas vezes prescritas pelo próprio médico para tratar sin-

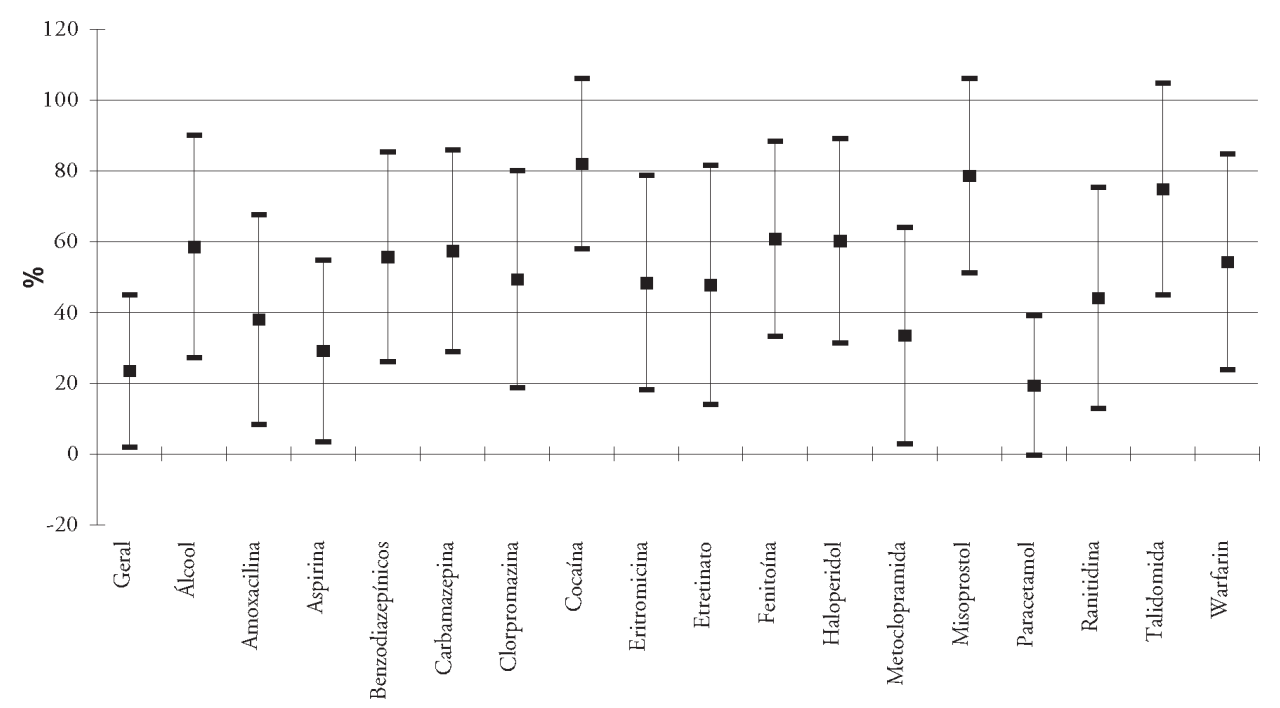

Figura 1 - Valores da média e desvio-padrão para a percepção de risco de teratogênese

Notas: As linhas denotam a média \pm 1 desvio-padrão da percepção do risco de teratogênese entre todos os entrevistados para as drogas pesquisadas. $\mathrm{O}$ retângulo hachurado indica o risco real estimado de teratogênese de cada droga. 
tomas comuns na gestação, pode ter influenciado na média da percepção de risco de tais drogas entre as gestantes, quando comparadas com as mulheres não grávidas.

Dentre os grupos que tiveram as menores taxas de acerto encontramos os estudantes iniciantes e as mulheres não gestantes, talvez por serem os participantes que estariam menos envolvidos com a gestação no momento da entrevista.

Acreditávamos que o acúmulo de conhecimento no decorrer do curso de medicina e na prática médica poderia melhorar a percepção dos riscos. No entanto, isto só foi observado em relação à amoxicilina, o haloperidol e a ranitidina, cujas médias dos riscos percebidos foram menores entre os clínicos, quando comparadas com as dos estudantes, o que demonstra menor receio na utilização de tais medicações. Por outro lado, em relação às outras drogas, as médias dos riscos percebidos continuaram superestimadas, principalmente de drogas com potentes efeitos teratogênicos, como a talidomida e o etretinato, sugerindo que informações científicas podem ser mal interpretadas, como foi salientado por outros autores (KOREN, 1998; KOREN; LEVICHEK, 2002). Esse efeito é minimizado com a prática clínica em relação às drogas mais comuns.

Ao compararmos as médias de percepção do risco dos ginecologistas e obstetras com as dos outros grupos, percebemos que os primeiros apresentaram médias mais próximas do risco real e estatisticamente diferente em relação à amoxicilina, ao benzodiazepínico, à cocaína, ao misoprostol, ao paracetamol e à ranitidina, e superestimada para o etretinato e a talidomida. Atribuímos esse fato de as primeiras medicações, diferentemente das últimas, serem mais comuns e freqüentemente prescritas ou proscritas por esses médicos.

De forma similar, divulgações não científicas em televisão, rádio e revistas populares podem levar à superestimação do risco real de drogas comuns entre os leigos, como foi salientado por alguns autores (KOREN, 2001; KOREN; LEVICHEK, 2002) e observado em nosso estudo entre as mulheres não gestantes, quando questionadas sobre o álcool, a cocaína, o haloperidol e o misoprostol. Por outro lado, drogas potencialmente teratogênicas, como a talidomida e o etretinato, mas pouco referidas nos meios de comunicação, podem ter tido média de risco próximo ao real entre os leigos, decorrente da subestimação do risco.

A percepção do risco real dos efeitos teratogênicos referentes às diferentes drogas às quais as mulheres estão expostas durante a gestação é de fundamental importância para que medidas errôneas, como a abstenção ao tratamento de doenças maternas crônicas ou agudas, ou uso inadvertido de drogas potencialmente teratogênicas, ou interrupção da gestação de fetos saudáveis não sejam adotadas, visto que tais condutas poderão ter repercussões dramáticas por toda a vida da criança e da família.

\section{CONCLUSÕES}

Através das observações deste estudo, verificamos que o risco real teratogênico é superestimado tanto pela população leiga como pelos médicos. Acreditamos que esse fato ocorra pela dificuldade de acesso às informações atualizadas.

Por outro lado, é importante salientar que constantemente novas drogas estão sendo lançadas no mercado e que o médico ou o leigo não tem obrigação de estar sempre bem informado sobre teratologia. Para obter tais informações, dispomos de serviços especializados como o SIAT (Serviço de Informação sobre Agentes Teratogênicos), que podem ser consultados por todos e precisam ser melhores divulgados. $\mathrm{O}$ SIAT-BA fornece orientaçôes para os leigos e pode ajudar os médicos na interpretação dos resultados.

Além disso, os meios de comunicação deveriam ser mais cautelosos, melhor orientados e mais fiscalizados por parte dos órgãos de saúde, em relação às divulgações não científicas sobre os riscos teratogênicos, visando a evitar repercussões danosas. 


\title{
Teratogenic risk: the perception in population different sections
}

\begin{abstract}
The wrong perception of the true teratogenic risk of the drugs used in pregnancy may lead the mistaken management of pregnancy: the unnecessary termination of the wanted pregnancy or damages fetuses forever. The knowledge about the teratogenic risk is very important for the general population, and mainly for the health professionals. The perception of the teratogenic risk of fifteen common medications and two illicit drugs by physicians, medical students and lay women was assessed employing a visualanalogue scale with the percentual risk of a child born with congenital anomaly caused by drugs used during pregnancy. The average of the teratogenic risk for the assessed drugs was higher than the true risk in all groups. The most common risk of malformation in the general population was recognised by gynaecologists and obstericians. The best improvement was with the more common drugs used in pregancy (paracetamol and amoxycillin) and recognised by gynaecologists and obstetricians.
\end{abstract}

Keywords: Teratogenic risk; pregnancy; genital anomaly.

\section{REFERÊNCIAS}

BATTINO, D. Assessment of teratogenic risk. Epilepsy Res., Amsterdam, v.45, p.171-173, 2001.

CEFALO, R.C. Drug labeling and risk perceptions of teratogenicity: a survey of pregnant Canadian women and their health professionals. Obstet. Gynecol. Surv., Baltimore, v.56, n.2, p.80-81, 2001.

JASPER, J.D. et al. Effects of framing on teratogenic risk perception in pregnant women. Lancet, London, v.358, p.1237-1238, 2001.

KOREN, G. Misrepresentation and miscommunication of teratogenic risk of drugs: analysis of three highly publicized international cases. Reprod. Toxicol., Elmsford, v.15, p.1-3, 2001.

KOREN, G.; LEVICHEK, Z. The teratogenicity of drugs for nausea and vomiting of pregnancy: perceived versus true risk. Am. J. Obstet. Gynecol., St. Louis, v.186, n.5, p.S248252, 2002.

KOREN, G.; PASTUSZAK, A.; ITO, S. Drugs in pregnancy. N. Engl. J. Med., Boston, v.338, n.16, p.1128-1137, 1998.
POLIFKA, J.E.; FAUSTMAN, E.M.; NEIL, N. Weighing the risks and the benefits: a call for the empirical assessment of perceived tertogenic risk. Reprod. Toxicol., Elmsford, v.11, n.4, p.633-640, 1997.

SANSEVERINO, M.T.V.; SPRITZER, D.T.; SCHÜLER-FACCINI, L. Manual de teratogênese. Porto Alegre: Ed. da Universidade, 2001.

SANZ, E.; GOMEZ-LOPEZ, T.; MARTINEZ-QUINTAS, M.J. Perception of teratogenic risk of common medicines. Eur. J. Obstet. Gynecol. Reprod. Biol., Limerick, v.95, p.127-131, 2001.

SCHARDEIN, J.L. Drug usage in pregnancy. In:____ (Org.) Chemically induced birth defects. NewYork: Marcel Dekker, 2000. p.6778 .

STREISSGUTH, A.P. et al. Aspirin and acetaminophen use by pregnant women and subsequent child IQ and attention decrements. Teratology, New York, v.35, n.2, p.211-219, 1987.

Recebido em / Received: 02/04/2005 Aceito em / Accepted: 07/07/2005 